\title{
Medical Management of Cesarean Scar Pregnancy at Latifa Hospital Dubai Health Authority, Dubai, UAE
}

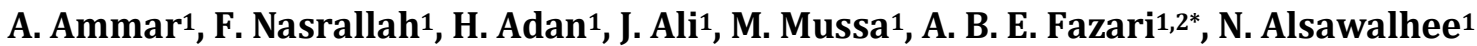 \\ ${ }^{1}$ Latifa Hospital for Women \& Children, DHA, Dubai, UAE \\ ${ }^{2}$ University of Medical Sciences \& Technology, Khartoum, Sudan \\ Email: *atiffazari@hotmail.co.uk
}

How to cite this paper: Ammar, A., Nasrallah, F., Adan, H., Ali, J., Mussa, M., Fazari, A.B.E. and Alsawalhee, N. (2020) Medical Management of Cesarean Scar Pregnancy at Latifa Hospital Dubai Health Authority, Dubai, UAE. Open Journal of Obstetrics and Gynecology, 10, 526-537. https://doi.org/10.4236/ojog.2020.1040047

Received: March 19, 2020

Accepted: April 17, 2020

Published: April 20, 2020

Copyright $(2020$ by author(s) and Scientific Research Publishing Inc. This work is licensed under the Creative Commons Attribution International License (CC BY 4.0).

http://creativecommons.org/licenses/by/4.0/ (c) (i) Open Access

\begin{abstract}
Introduction: Cesarean scar pregnancy (CSP) is defined as implantation of gestational sac at the site of cesarean scar. It's a serious diagnosis that has become more prevalent in recent years and related to the increasing rate of cesarean sections reported worldwide. Identifying these cases and treating them is challenging, with no agreed upon universal protocol for successful treatment. We aim to evaluate the success rate and outcome of medical management for Cesarean scar pregnancy. Methods: It was a retrospective descriptive study of all cesarean scar pregnancies managed at fetal medicine unit at Latifa Hospital in Dubai, UAE the main obstetrics \& Gynecology tertiary hospital in Dubai Emirate, UAE from 2015 to 2017. Certainly, a set of diagnostic criteria were implemented to confirm the diagnosis of CSP. The cases were then offered our proposed management which is systemic methotrexate injections \pm KCL. Follow up made by serial $\beta$ hcg and ultrasound scan. The data were collected used specified data collection sheet for this purpose then analyzed and presented using statistical package for social sciences (SPSS) version 26. Results: 33 cases of cesarean scar pregnancies are confirmed and medically managed during the study period. The study subjects composed of a group of patients who had a viable CSP and received local KCL injection + systemic methotrexate, a second group who had non-viable CSP and received systemic methotrexate 20 cases were viable pregnancies who received combined local potassium chloride plus systemic methotrexate, and 13 were non-viable received systemic methotrexate only. The mean gestational age at diagnosis was 8 weeks ( $\mathrm{SD} \pm 1.8$ ). On average our cases had a history of 3 previous cesarean sections (range $1-6$ ). Overall, the success rate calculated from our study population for medical management of CSP was $77.8 \%$; this varied between viable CSP which had a success rate of $66.7 \%$ and non-viable
\end{abstract}


CSP which had a success rate of $100 \%$. The average period of outpatient follow-up for the patients to achieve complete resolution was 14 weeks (SD \pm 7.5). Conclusions: Medical management of CSP in the form of systemic methotrexate \pm local KCL injections proves to have acceptability and a good success rate especially for non-viable CSP, low complications rate and with the benefit of preserving future fertility.

\section{Keywords}

Caesarean Scar Pregnancy, Methotrexate, Potassium Chloride, Maternal Morbidity

\section{Introduction}

Cesarean scar pregnancy (CSP) is a form of ectopic pregnancy. Its incidence is increasing over the last few years and it doesn't appear to correlate with the number of cesarean deliveries.

The pregnancy sac is either fully or partially implanted within the scar of previous cesarean section or hysterotomy, and it is surrounded by myometrial tissue. The incidence is around 1 in 2000 of all pregnancies [1] [2].

Around $6 \%$ of ectopic pregnancies among women with at least one previous cesarean scar will be diagnosed as cesarean scar pregnancy [3] [4].

The first case of cesarean scar pregnancy reported in English medical literature was in 1978. Until 2001 only 190 cases were reported. However, this number significantly increased to reach more than 1000 cases over the following years. This could be due to the increase in numbers of repeated cesarean sections, increased awareness about the morbidities related to this condition and the importance to detect them early so they can be managed more safely with the best possible outcome for the patient. On the other hand, the marked improvement in the quality of the ultrasound imaging also played a role in the accurate detection of these cases.

The natural history of this condition evolves quickly in a few weeks. Therefore, if allowed to continue, complications can occur including rupture of the cesarean scar with life-threatening hemorrhage. This, in turn, may warrant life-saving hysterectomy and loss of future fertility. Hence, many of these cases once diagnosed will be terminated in the $1^{\text {st }}$ trimester to avoid the expected complications associated with this type of pregnancy. On the contrary, pregnancies which continue will mostly present with morbidly adherent placenta in later gestation, which is by itself a high-risk pregnancy and can end by massive obstetric bleeding and hysterectomy. Since 1985 cesarean section rates have been on the rise both in developed and developing countries [1] deeming it necessary procedure in many cases to reduce both maternal and fetal morbidity and mortality [5] [6].

The cesarean section rate in the UAE has been estimated at $9.3 \%-15.2 \%$ in 
the past decade although recent accurate data is not available [4] [5]. However, the cesarean section in our tertiary hospital is around $30 \%$. Along with this rise came a number of morbidities for patients who had cesarean deliveries like morbidly adherent placenta (MAP) and cesarean section scar pregnancies (CSP). The rate of MAP (accreta, increta, and percreta) has increased slowly from 1 in 30,000 deliveries in the 1930s to 1 in $2000-3000$ deliveries in the last 10 years or so [6] [7] [8]. In addition, as the number of previous Cesarean deliveries increases, so does the risk of faulty placentation [6]. Both pathologies carry the same pathogenesis and most likely represent a continuum. Similarly, we have noticed a rise in these complications in our facility.

\section{Pathophysiology:}

The primary cause of cesarean scar pregnancy is still not clear; however, its occurrence may be related to the presence of an existing scar defect, or dehiscent tract formed between the previous scar and the endometrial cavity [9].

Those scars will have a global effect on the endometrial cavity preventing the pregnancy to implant in a normal position. Whether they are previous cesarean section scars, or trauma to the endometrium by other uterine surgeries like curettage, hysteroscopy, or myomectomy. This causes damage of the decidua basalis leading to persistence of tiny dehiscent tracts or small wedge defects in which the CSP will implant [10].

The risk of scar pregnancy could be proportional to the size of the uterine wall defect as it was noticed that patients who had cesarean section for breech presentation were at higher risk of developing scar pregnancy; and theoretically it could be due to large defects resulting from higher uterine incisions in poorly formed lower segments [8].

Two types of cesarean scar pregnancy were described by Vial et al. [10]. The classification is based on the ultrasound findings and pregnancy progression. The first type is when the gestational sac grows towards the endometrial cavity and is called the endogenic type. The other type or the exogenous type is when the pregnancy sac is deeply implanted in the scar area and surrounded by the myometrial tissue and the progress of the sac is towards the bladder and abdominal cavity causing thinning of the myometrial layer between the sac and the bladder. The sac will bulge through the scar and eventually have a high risk of earlier rupture and a higher risk of intraabdominal hemorrhage in earlier gestation [8] [10].

Although the incidence of morbidly adherent placenta was related directly with the increase in the number of cesarean sections, it is not the same for the cesarean scar pregnancy.

So far there is no convincing evidence in the literature if the technique of closure of the uterine incision during the cesarean section, whether a single- or double-layer closure, has any role to put the patient at risk for a cesarean scar pregnancy. Furthermore, it could not be proven whether the occurrence of CSP was increased in a recent scar versus an old scar because cesarean scar pregnancies were reported in both scenarios. 
The risk of recurrence was reported as $3.2 \%$ to $5.0 \%$ in women with a history of previous cesarean scar pregnancy treated by dilatation and curettage with or without uterine artery mobilization [8].

Risk factors observed to be associated with recurrence included the presence of a thin anterior uterine wall at the site of the previous scar of less than $5 \mathrm{~mm}$, previous scar pregnancy of the exogenous type where the sac was bulging into the uterovesical fold, previous delivery in a low community hospital [8].

The recurrence of cesarean scar pregnancy is also reported after surgical repair of the scar area although theoretically it was thought to decrease the recurrence. This indicates the need for more research to find the ideal way for surgical repair of a uterine niche to prevent recurrence of cesarean scar pregnancies [8] [11].

\section{Presentation:}

Many cases (as high as $37 \%$ ) are asymptomatic and are suspected during a routine ultrasound in early pregnancy. Symptomatic patients can vary in their presentations between early painless vaginal bleeding in 39\% of cases, $16 \%$ will present with moderate abdominal pain, and $9 \%$ will present with mild abdominal pain. Severe abdominal pain with profuse bleeding indicate impending rupture, and if the patient presented with hypovolemic shock will indicate rupture scar with intraabdominal bleeding [8] [10].

\section{Diagnosis:}

The use of ultrasound imaging is the first line tool for the diagnosis of cesarean scar pregnancies [8] [12].

Transabdominal and transvaginal ultrasound are combined to reach a more accurate diagnosis. Using three-dimensional imaging is not necessary but may complement the 2D scan findings.

The typical steps in diagnosis start by having a high index of suspicion. Ultrasound shows that the pregnancy sac is implanted in the lower uterine segment surrounded by myometrial tissue with empty and closed upper endometrial cavity, and empty cervical canal.

The shape of the gestational sac can vary according to the gestation age and type of scar pregnancy. So it can be triangular in shape in early pregnancy and becomes oval in shape as pregnancy progresses. It can be associated with a yolk sac and viable pregnancy like a normal pregnancy [10] [13]. Using color, Doppler will show a high vascularity flow around the gestational sac, especially at the myometrial bladder interface with thinned out myometrium or complete absence of myometrium [10] [13] [14].

When we do the ultrasound, an effort should be made to rule out other differential diagnoses like inevitable miscarriage with a low-lying sac or cervical pregnancy.

The early stages of miscarriage can look like a scar pregnancy but usually will be irregular, present within the endometrial cavity, with less or absent vascularity, and will have a positive sliding sign. Adding MRI can be useful to clear out 
the diagnosis in certain conditions when the ultrasound features are not clear [8].

\section{Management:}

Once the diagnosis is established, the patient needs to be explained in detail and counseled regarding the options of management. The aim of the treatment option should be towards ending the pregnancy as soon as the diagnosis is established and to remove the gestational sac from the scar area to preserve future fertility.

Different options for treatment are available. Unfortunately, no standardized protocol or guideline is available for an ideal treatment. In Latifa Hospital, we formulated our protocol for the management of cesarean section scar pregnancy after reviewing what was published in the literature and our personal experience in treating many of the cases which were referred to our unit. The treatment options given should be individualized according to the patient's clinical presentation, the stability of the patient, and the experience of the staff available to treat the condition whether it is a medical approach or a surgical approach or both if needed.

The choice of treatment can vary between conservative expectant management which is not usually recommended because of the major complication associated with the condition or medical management in medically and hemodynamically stable patients with mild or no symptoms. In this type of treatment, Methotrexate is the best drug used and has a good success rate with pregnancies below 8 weeks and with the HCG levels of less than 5000 IU. It can also be offered as combined local and systemic methotrexate with sac aspiration for a better success rate. On the other hand, surgical options should be offered to a patient with significant symptoms or who refused and declined medical treatment. Furthermore, the type of surgical treatment should be chosen according to the type of pregnancy whether it's an exogenous or endogenous scar pregnancy, the myometrial thickness and the experience of the team managing the case. In certain occasions, different treatment combination can be considered if one modality failed to have complete resolution of the pregnancy, or the process of the resolution was prolonged.

\section{Objectives:}

Our main objective was to evaluate the success rate and outcomes of medical management in cesarean scar pregnancies diagnosed in the $1^{\text {st }}$ trimester. We also aimed to determine the total period of follow up for the study subjects until complete resolution to assess feasibility of our proposed management for both patients and the hospital.

\section{Material \& Methods}

This is a retrospective descriptive review done at Latifa Hospital the main tertiary care for obstetrics and Gynecology at Dubai Emirates, United Arab Emirates. It has large catchment area involves the Northern Emirates specially for 
the high risk cases the annual registered deliveries are $2015=3950,2016=4123$ and $2017=4760$ where the total accepted and managed cases is 34,236 cases. It is well equipped hospital runs by consultants led teams in all specialties and subspecialties.

During the study period the hospital accepted 33 cases diagnosed cases of CSP, all were reviewed, diagnosed and managed during the study period of January 2015 to December 2017.

We followed these patients at the fetal medicine unit according to a set of diagnostic criteria [11] [12] as fellow:

1) Empty endometrial cavity with a gestational sac between the cavity and cervix.

2) Positive color Doppler of blood vessels surrounding the gestation sac.

3) Gestational sac embedded in the scar (thin myometrium of $1-3 \mathrm{~mm}$ or less)

4) Triangular shape of the sac in the sagittal plane in those pregnancies that were around 5 weeks.

5) A prominent vascular area in the site of a previous cesarean delivery scar.

6) Positive serum beta human chorionic gonadotrophin ( $\beta$-HCG).

All of them were counseled to receive medical management initially, and surgical management would only be resorted to if needed like in the presence of massive bleeding or signs of rupture.

In case of a viable CSP a combination of local/intra-saccular KCL injection and methotrexate single or multiple doses was offered. In non-viable CSP systemic methotrexate single or multiple doses was offered as a mode of management for these patients.

Subsequently, the patients were followed up with weekly $\beta$-HCG readings and ultrasound scans. The aim of the scans was to measure the size of the gestational sac and to subjectively assess the vascularized area for 3 consecutive weeks. Thereafter scans were done fortnightly until $\beta$-HCG was undetectable and near complete resorption of the products of conception was achieved. Complete resolution was defined as a negative BHCG and the size of the retained products of conception of $<2 \mathrm{~cm}$ with no vascularity.

The data collected was then analyzed using Statistical Package for Social Studies (SPSS) version 25 in order to find any statistically significant findings.

\section{Results}

There were 44 patients referred to our fetal medicine unit as suspected CSP during our study period. Out of these, 33 met the ultrasound diagnostic criteria of CSP which we have set as a guideline in our unit after reviewing current available literature.

Out of the 33 CSP cases, 20 were viable pregnancies and 13 were non-viable pregnancies (Table 1). One of the cases from the non-viable group defaulted so for the sake of discussion was removed from the analysis of the outcomes. 
Table 1. The intervention done for CSP.

\begin{tabular}{|c|c|c|}
\hline & VIABLE $\mathrm{n}=20$ & NON-VIABLE $\mathrm{n}=12$ \\
\hline \multicolumn{3}{|l|}{ Initial treatment: } \\
\hline$-\mathrm{KCL}+\mathrm{MTX}$ & 17 & - \\
\hline - KCL only & 1 & - \\
\hline - MTX & - & 9 \\
\hline - Expectant & 2 & 3 \\
\hline $\begin{array}{l}\text { Need for surgical intervention after medical } \\
\text { management }\end{array}$ & $6(33.3 \%)$ & $0(0 \%)$ \\
\hline Success/complete resolution & $12(66.7 \%)$ & $9(100 \%)$ \\
\hline Time to resolution & Mean 14.2 weeks & Mean 15.7 weeks \\
\hline
\end{tabular}

The mean age of our patient was 37 years $(\mathrm{SD} \pm 4.3)$ and their mean parity was 3 . On average our cases had a history of 3 previous cesarean sections (range 1 - 6). The mean gestational age at diagnosis was 8 weeks ( $\mathrm{SD} \pm 1.8$ ).

Mostly the cases presented to our center with complaints of vaginal bleeding and/or pain $-67 \%$. The rest $-34 \%$ were referred from the private sector. The mean BHCG range was 41,771 at diagnosis and the values ranged from 1178 to 162,573 .

As per our hospital protocol, all the viable pregnancies were offered fetal intra-cardiac KCL injections or inside the gestational sac (intra-saccular) followed by intramuscular methotrexate injection. On the other hand, the non-viable CSP's were offered intramuscular methotrexate injections.

Overall, the success rate calculated from our study population for medical management of CSP was $77.8 \%$, but there was a difference in the success rate when comparing viable to non-viable cesarean scar pregnancies as detailed below (Table 1).

17 out of the 20 viable CSP (90\%) opted for our proposed management and received Potassium chloride and Methotrexate (KCL + MTX). One case was a twin pregnancy with one viable scar pregnancy and after counseling opted for KCL injection in the CSP sac. However, two cases opted for conservative management.

Among the cases who received medical management in the form of MTX \pm KCL, 12 patients had complete resolution of the CSP on follow up indicating a $66.7 \%$ success rate for this proposed management in our study population (Table 2). Among the remainder of the patients, 33.3\% needed surgical intervention for medical reasons. Five cases had ERPOC under USS guidance due to heavy bleeding ( 4 cases) and 1 case due to sepsis. One case had a hysteroscopic resection of RPOC due to the persistence of the CSP and continued bleeding which was bothersome to the patient (Figure 1 and Figure 2).

One of the 2 cases who opted for conservative management continued her pregnancy and consequently had a placenta accreta which ended in a cesarean hysterectomy. The second viable CSP who opted to continue her pregnancy after counseling had an elective cesarean section at term and had partial placenta accreta associated with a bladder injury. 
Table 2. The outcomes of viable CSP.

\begin{tabular}{|c|c|c|c|c|c|c|}
\hline No & Maternal Age & GA & PCD & Treatment & Clinical Course & Complications \\
\hline 1 & 27 & 8 & 2 & KCL + MTX & Resolution & Mouth ulcers \\
\hline 2 & 30 & 8 & 1 & $\mathrm{KCL}+\mathrm{MTX}$ & Resolution & - \\
\hline 3 & 30 & 9 & 1 & KCL + MTX & ERPOC & Bleeding \\
\hline 4 & 36 & 9 & 3 & $\mathrm{KCL}+\mathrm{MTX}$ & Resolution & - \\
\hline 5 & 36 & 10 & 3 & Expectant & Partial Accreta & Bladder injury \\
\hline 6 & 36 & 10 & 4 & $\mathrm{KCL}+\mathrm{MTX}$ & ERPOC & Bleeding \\
\hline 7 & 37 & 6 & 6 & $\mathrm{KCL}+\mathrm{MTX}$ & Resolution & - \\
\hline 8 & 40 & 9 & 3 & $\mathrm{KCL}+\mathrm{MTX}$ & Resolution & - \\
\hline 9 & 44 & 9 & 5 & $\mathrm{KCL}+\mathrm{MTX}$ & Resolution & - \\
\hline 10 & 44 & 11 & 2 & $\mathrm{KCL}+\mathrm{MTX}$ & Resolution & - \\
\hline 11 & 41 & 11 & 3 & $\mathrm{KCL}+\mathrm{MTX}$ & ERPOC & Sepsis \\
\hline 12 & 42 & 8 & 2 & $\mathrm{KCL}+\mathrm{MTX}$ & Resolution & - \\
\hline 13 & 37 & 8 & 2 & Expectant & Accreta & CS hystertomy \\
\hline 14 & 39 & 9 & 4 & KCL + MTX & Hysteroscopic resection & - \\
\hline 15 & 37 & 12 & 4 & $\mathrm{KCL}+\mathrm{MTX}$ & Resolution & - \\
\hline 16 & 39 & 8 & 4 & $\mathrm{KCL}+\mathrm{MTX}$ & Resolution & - \\
\hline 17 & 34 & 7 & 3 & $\mathrm{KCL}+\mathrm{MTX}$ & Resolution & - \\
\hline 18 & 32 & 10 & 4 & $\mathrm{KCL}+\mathrm{MTX}$ & ERPOC & - \\
\hline 19 & 34 & 7 & 2 & KCL in 1 fetus & Resolution & - \\
\hline 20 & 34 & 9 & 4 & $\mathrm{KCL}+\mathrm{MTX}$ & ERPOC & - \\
\hline
\end{tabular}

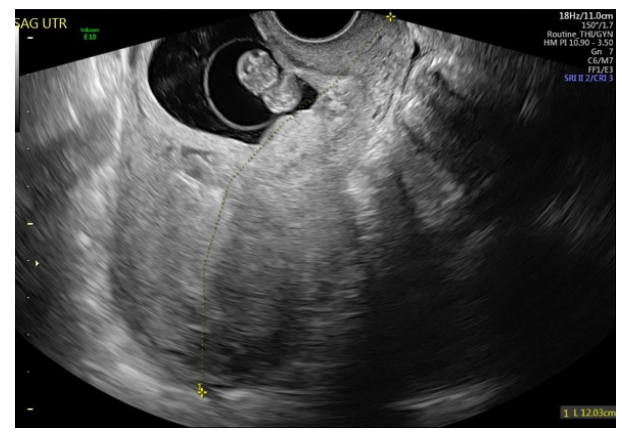

Figure 1. Viable cesarean scar pregnancy before receiving treatment.

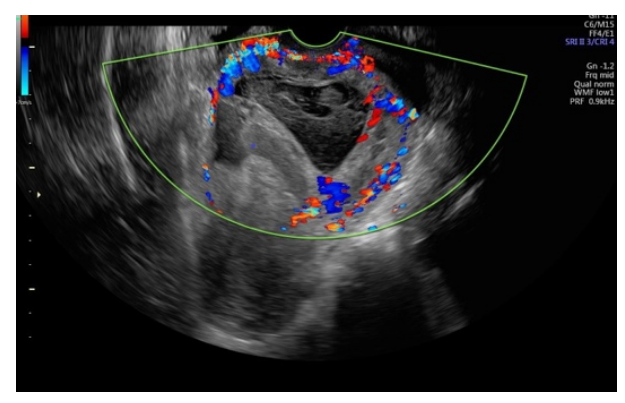

Figure 2. Cesarean scar pregnancy after receiving treatment; still showing high vascularity. 
With reference to the 12 non-viable CSP in our study, 9 patients (75\%) opted for systemic MTX injections, 3 cases only wanted expectant management after explaining possible risks. Among those who opted for methotrexate injections, all of them had complete resolution indicating a $100 \%$ success rate for this modality of treatment for non-viable CSP in our study population. Two of those who opted for expectant management also have a complete resolution; however, one case underwent ERPOC under USS guidance due to heavy bleeding (Table 3).

The rate of complications in our study population was relatively low. Among the 26 patients who had received methotrexate, there were 2 cases $(6 \%)$ who had mouth ulcerations. Heavy vaginal bleeding was the more common complication in 6 patients in total (18\%) underwent ERPOC to control the heavy bleeding.

The average period of outpatient follow-up for the patients to achieve complete resolution was 14 weeks ( $S D \pm 7.5$ ). $90.9 \%$ of our cases had a total follow up period of $\leq 24$ months. Only 3 cases had a follow-up period of $>24$ months (Figure 3).

Table 3. The outcomes of non-viable CSP.

\begin{tabular}{ccccccc}
\hline No & Maternal Age & GA & PCD & Treatment & Clinical Course & Complications \\
\hline $\mathbf{1}$ & 40 & 7 & 3 & Expectant & ERPOC & Bleeding \\
$\mathbf{2}$ & 31 & 6 & 1 & Expectant & Resolution & - \\
$\mathbf{3}$ & 31 & 6 & 2 & MTX & Resolution & - \\
$\mathbf{4}$ & 35 & 8 & 4 & MTX & Resolution & - \\
$\mathbf{5}$ & 35 & 6 & 1 & MTX & Resolution & - \\
$\mathbf{6}$ & 35 & 6 & 1 & MTX & Resolution & - \\
$\mathbf{7}$ & 37 & 6 & 4 & MTX & Resolution & - \\
$\mathbf{8}$ & 39 & 11 & 6 & MTX & Resolution & - \\
$\mathbf{9}$ & 39 & 7 & 1 & MTX & Resolution & - \\
$\mathbf{1 0}$ & 40 & 8 & 2 & MTX & Resolution & - \\
$\mathbf{1 1}$ & 42 & 6 & 1 & MTX & Resolution & Mouth ulcers \\
$\mathbf{1 2}$ & 42 & 7 & 4 & Expectant & Resolution & - \\
\hline
\end{tabular}

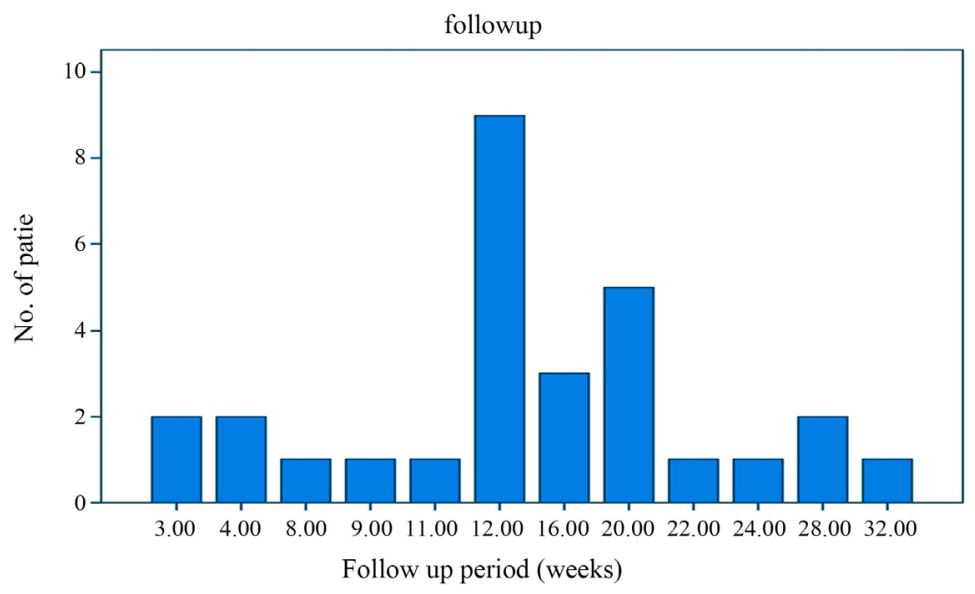

Figure 3. The numbers of weeks needed for the follow up for of CSP patients. 


\section{Discussion}

Latifa hospital accepted 34,236 cases out of them 33 cases are CSP that make rate of CSP is $0.096 \%$ which is double the reported rate $0.04 \%-0.05 \%$ [15]. Medical management of CSP with MTX \pm KCL is quite an effective method in treating this condition. This method is minimally invasive, with fewer complications and has high chance of preserving fertility [13] [14].

The success rate was almost $78 \%$. However, it is important to notice that medical management was significantly more successful in non-viable CSP than viable pregnancies. In our study population, we achieved a $100 \%$ success rate with medical management for non-viable CSP. On the other hand, medical management for viable CSP had a success rate of $67 \%$ which translates into a failure rate of around $23 \%$ or in other words $1 / 3$ of the cases ended up needing further surgical intervention. We still opted for more conservative surgical interventions in our patients such as hysteroscopic resection to maintain her future fertility.

Our findings are in agreement with a case series published in 2012 were 19 out of their 26 patients received medical management in the form of MTX inside the gestational sac as well as intra-muscular, and they all achieved complete resolution with no complications [13]. They had a slightly different protocol than ours in that we do not use intra-saccular cytotoxic agent unless it's a viable pregnancy. Moreover, a literature review in 2015 which included all reports of CSP treated medically found a success rate of $73.9 \%$ after a single local methotrexate injection and an accumulated success rate of $88.5 \%$ if multiple dose protocol of methotrexate is used [15].

Likewise, another published report of 26 cases collected between 2009 \& 2015 found that most of their cases were treated successfully by medical management in the form of methotrexate and they noticed a higher success rate with local or intra-saccular MTX injection more than systemic injections (93.75\% vs $73.33 \%$ ). However, their mean BHCG level was 20,610 while in our study population the mean baseline BHCG level was 41,771 . This may explain their higher success rate [15]. Still, this information warrants further contemplation and research into whether local injection in viable or a non-viable CSP really increases the success rate of this modality of treatment and so far, some literature seems to be pointing in that direction.

In regard to the mean follow up period, in our study, it was around 14 weeks and this is comparable to studies like ours which reported a follow-up period of 12.5 weeks on average [11].

\section{Conclusions}

Cesarean scar pregnancy is a significant pathology with increasing prevalence in current practice. The early diagnosis and management of this condition is crucial to reduce the severe complications related to this type of pregnancy. Most of the literature emphasize early intervention and termination of pregnancy; still 
there is no global agreement about the best preferred type of management. Using the medical treatment option specially combining the locally injected methotrexate with the systemic methotrexate injections is a less invasive method with satisfactory results.

Further research on possible direct predisposing factors in trying to prevent the occurrence of cesarean scar pregnancies is of utmost importance. One of the most obvious action plans is to reduce the overall number of cesarean sections to stop the sequelae of this condition in the future. In addition, clinicians should always have a high index of suspicion in all cases with a previous cesarean scar to diagnose cases of CSP at the earliest possible gestation before high BHCG levels are reached so that their management is simpler and medical management more likely to succeed.

\section{Constrains}

This is a retrospective study, numbers of limitation concerning the data collection for such diagnosis is the main issue as the data is from the files and some are missed. Our unit is unique newly established unit still some policies and guidelines need retuning and to be well adopted in our practice. More promotion and counseling for the cases and referral system are need.

\section{Conflicts of Interest}

The authors declare no conflicts of interest regarding the publication of this paper.

\section{References}

[1] WHO (2015) WHO Statement on Caesarean Section Rates.

[2] Jurkovic, D., Hillaby, K., Woelfer, B., Lawrence, A., Salim, R. and Elson, C.J. (2003) First Trimester Diagnosis and Management of Pregnancies Implanted into the Lower Uterine Segment Cesarean Section Scar. Ultrasound in Obstetrics \& Gynecology, 21, 220-227. https://doi.org/10.1002/uog.56

[3] Luke, R.K., Sharpe, J.W. and Greene, R.R. (1966) Placenta Accreta: The Adherent or Invasive Placenta. American Journal of Obstetrics \& Gynecology, 95, 660-668. https://doi.org/10.1016/S0002-9378(16)34741-X

[4] Wu, S., Kocherginsky, M. and Hibbard, J.U. (2005) Abnormal Placentation: Twenty-Year Analysis. American Journal of Obstetrics \& Gynecology, 192, 1458. https://doi.org/10.1016/j.ajog.2004.12.074

[5] Molinaro, T. and Barnhart, K. (2019) Abdominal Pregnancy, Cesarean Scar Pregnancy, and Heterotopic Pregnancy. https://Uptodate.com

[6] Jayaram, P., Okunoye, G. and Konje, J. (2017) Caesarean Scar Ectopic Pregnancy: Diagnostic Challenges and Management Options. The Obstetrician \& Gynecologist, 19, 13-20. https://doi.org/10.1111/tog.12355

[7] Seow, K., Huang, L., Lin, Y., Yan-Sheng Lin, M., Tsai, Y. and Hwang, J. (2004) Cesarean Scar Pregnancy: Issues in Management. Ultrasound in Obstetrics \& Gynecology, 23, 247-253. https://doi.org/10.1002/uog.974

[8] Ash, A., Smith, A. and Maxwell, D. (2007) Caesarean Scar Pregnancy. BJOG: An 
International Journal of Obstetrics \& Gynaecology, 114, 253-263.

https://doi.org/10.1111/j.1471-0528.2006.01237.x

[9] Timor-Tritsch, I., Monteagudo, A., Santos, R., Tsymbal, T., Pineda, G. and Arslan, A. (2012) The Diagnosis, Treatment, and Follow-up of Cesarean Scar Pregnancy. American Journal of Obstetrics and Gynecology, 207, 1-13. https://doi.org/10.1016/j.ajog.2012.04.018

[10] Timor-Tritsch, I., Khatib, N., Monteagudo, A., Ramos, J., Berg, R. and Kovács, S. (2015) Cesarean Scar Pregnancies: Experience of 60 Cases. Journal of Ultrasound in Medicine, 34, 601-610. https://doi.org/10.7863/ultra.34.4.601

[11] Timor-Tritsch, I., Monteagudo, A. and Goldstein, S. (2014) How to Identify and Manage Cesarean-Scar Pregnancy. OBG Management, 26, 19-27.

[12] Kanat-Pektas, M., Bodur, S., Dundar, O. and Lale Bakır, V. (2016) Systematic Review: What Is the Best First-Line Approach for Cesarean Section Ectopic Pregnancy? Taiwanese Journal of Obstetrics \& Gynecology, 55, 263-269. https://doi.org/10.1016/j.tjog.2015.03.009

[13] Timor-Tritsch, I.E. and Monteagudo, A. (2012) Unforeseen Consequences of the Increasing Rate of Cesarean Deliveries: Early Placenta Accreta and Cesarean Scar Pregnancy. A Review. American Journal of Obstetrics \& Gynecology, 207, 14-29. https://doi.org/10.1016/j.ajog.2012.03.007

[14] Cheung, V.Y. (2015) Local Methotrexate Injection as the First-Line Treatment for Cesarean Scar Pregnancy: Review of the Literature. The Journal of Minimally Invasive Gynecology, 22, 753-758. https://doi.org/10.1016/j.jmig.2015.04.008

[15] Kim, Y.R. and Moon, M.J. (2018) Ultrasound-Guided Local Injection of Methotrexate and Systemic Intramuscular Methotrexate in the Treatment of Cesarean Scar Pregnancy. Obstetrics \& Gynecology Science, 61, 147-153.

https://doi.org/10.5468/ogs.2018.61.1.147 